\title{
Bariatric Surgery Provided by the Veterans Health Administration: Current State and a Look to the Future
}

\author{
William Gunnar, MD, $J D^{1,2}$ \\ ${ }^{1}$ Veterans Health Administration, Washington, DC, USA; ${ }^{2}$ The George Washington University, Washington, DC, USA.
}

J Gen Intern Med 32(Suppl 1):S4-S5

DOI: $10.1007 / \mathrm{s} 11606-017-3991-4$

(c) Society of General Internal Medicine 2017

$\mathrm{T}$ he Veterans Health Administration (VHA) provides integrated health care and treatment for 8.5 million enrolled Veterans through a regional system of care defined by 18 Veterans Integrated Service Networks (VISNs). VISNs are variable in geographic size and the number of operating VHA Surgery Programs (range 6-10 per VISN). In FY 2015, 135 VHA Surgery Programs performed 408,137 surgical procedures in 844 equipped operating rooms. During this same period, the VHA performed 453 bariatric surgery procedures.

Management of the overweight and obese patient is complex. A quick internet search identifies myriad options including diet, exercise, behavioral modification, mental health and substance abuse if needed, pharmacotherapy, acupuncture, intra-gastric balloon placement, and bariatric surgery. Approximately $78 \%$ of the Veteran population, or 6.6 million Veterans enrolled in the Department of Veterans Affairs (VA), are overweight or obese, of which $165,000(2 \%)$ are morbidly obese with a body mass index of $>40 .{ }^{1}$ Utilizing available national estimates, the number of bariatric surgery procedures performed by the VHA appears woefully low for the patient population. The American Society for Metabolic and Bariatric Surgery estimates that 196,000 bariatric surgery procedures were performed in 2015 . $^{2}$ For an at-risk US population of 80 million obese adults, an estimated rate of bariatric surgery would be 1 in every 400 at-risk patients. The rate for bariatric surgery in the VA is 1 in 14,500 overweight or obese Veterans.

So why is the rate of bariatric surgery in the VA lower than the US rate for an at-risk population? Potential reasons include: differing patient demographics, limited availability of services, and barriers to referral. Also important is the absence of a standardized plan of care grounded in evidence-based medicine and reflected in clinical decision support, care coordination agreements, and standardized metrics for assessing outcomes for successful care and treatment.

The VA population is older and predominately male. ${ }^{3} \mathrm{~A}$ review of the demographics and outcomes of the patients that underwent bariatric surgery in FY 2015 at a VHA Surgery

Published online March 7, 2017
Program indicate that the outcomes are excellent despite a population of older males with a significant rate of comorbid conditions. Specifically, those Veterans undergoing bariatric surgery are predominantly male (69\%) with an average age of 52 years and a high prevalence of diabetes (45\%), hypertension $(76 \%)$, and known heart disease $(9 \%)$. All the procedures performed (9 laparoscopic band, 129 Roux-en-Y, and 315 gastric sleeve) underwent risk assessment and outcomes analysis by the VHA National Surgery Office's VA Surgical Quality Improvement Program (VASQIP), recording no deaths at 30 days and a low complication rate $(0.0 \%$ laparoscopic band, 3.9\% Roux-en-Y, and 1.0\% gastric sleeve).

Regarding access, not all 135 VHA Surgery Programs have the infrastructure and approval to perform bariatric surgery procedures. In 2010, VHA Directive 2010-018, Facility Infrastructure Requirements to Perform Standard, Intermediate, or Complex Surgical Procedures, was published requiring each of the VHA Surgery Programs to be designated by the established facility infrastructure. Bariatric surgery procedures are designated as inpatient intermediate procedures requiring them to be relegated to the 98 VHA Surgery Programs designated as either inpatient intermediate (30) or inpatient complex surgery (68). In addition, VHA Directive 1043, Restructuring of VHA Clinical Programs, mandates that any VHA Surgery Program requesting to add bariatric surgery services must undergo a rigorous evaluation, including a business plan review and site visit of the facility by subject matter experts, prior to final clinical program approval by the VHA Under Secretary for Health.

In effect, through policy, each VISN determines the clinical needs of the enrolled Veterans it serves, resources the VHA Surgery Programs accordingly, and establishes specific programs based on a strategic plan. In FY 2015, the VHA had 21 VHA Surgery Programs across the country with approved bariatric surgery programs; however, these programs were not evenly distributed geographically or across VISNs. One VISN had 3 VHA-approved bariatric surgery programs, five VISNs had 2 programs, eight VISNs had 1 program, and four VISNs did not have a single approved bariatric surgery program.

Federal regulation establishes that the VA clinician is authorized to deliver the medical care and services needed to promote, preserve, or restore the health of the individual, which is consistent with generally accepted standards of medical practice (38 CFR §17.38). Complex decisions regarding who is best served by bariatric surgery, what the optimal 
preoperative work-up should be, and the parameters for postoperative follow-up are not standardized in practice across the organization. A recent questionnaire sent by the VHA National Surgery Office to the 21 VHA Surgery Programs approved to perform bariatric surgery identified variation in care and treatment outcomes including: the duration of participation in a preoperative weight loss program, the need to complete a preoperative weight loss program in all circumstances, the amount of weight loss required in a preoperative weight loss program, the percentage of patients referred for bariatric surgery that actually undergo the procedure, and the duration of postoperative follow-up and monitoring of outcomes.

It is worth noting that the VA is not limited to providing health care services to only the VHA Surgery Programs providing bariatric surgery. Under Title 38 USC $\$ 1703$, the VA has the authority to refer enrolled Veterans to a non-VA provider ("Community Care") when care and treatment are not feasibly available at a VA health care facility. ${ }^{4}$ The enactment of the Veterans Access, Choice and Accountability Act of 2014 (Pub. L. 113-146) extended Community Care to Veterans waiting more than 30 days for services, living greater than 40 miles from a VA health care facility, or experiencing an undue burden in receiving VA treatment and services. ${ }^{5}$ In FY 2015, the VA provided reimbursement to non-VA providers for 233 enrolled Veterans referred to Community Care for bariatric surgery. All VISNs referred Veterans for bariatric surgery under Community Care (range 3-32 procedures per VISN) regardless of the presence or absence of an approved VHA bariatric surgery program in the VISN.

The importance of the VA Health Services Research and Development (HSR\&D) sponsored Weight Management State of the Art (SOTA) conference held in March 2016 cannot be overstated. The recommendations of the Bariatric Surgery Workgroup (BSWG) will stimulate future research to guide standardized referrals of the at-risk population to bariatric surgery when appropriate, provide the foundation for standardizing care and treatment before, during, and after bariatric surgery based on the body mass index and clinical condition of the patient, and establish a framework for tracking and monitoring a standard set of intermediate and long-term functional outcomes for enrolled Veterans identified as being overweight or obese and those Veterans who have undergone bariatric surgery whether at a VHA Surgery Program or by a non-VA community provider.

The VA, which is the largest integrated health care system in the US, is well positioned and resourced to provide comprehensive weight management to the beneficiary population. Standardizing care and treatment of the overweight and obese Veteran demand an organizational strategy for care and treatment. The HSR\&D SOTA conference will establish a foundation for this strategy. Emerging knowledge will shape the guidelines for bariatric surgery and preoperative management to potentially include pharmacotherapy, behavior modification, and temporary intragastric balloon placement. The VA currently provides bariatric surgery at 21 VHA Surgery Programs approved to perform bariatric surgery and through nonVA community providers when appropriate. If and when referrals for bariatric surgery increase, the VA has an established process to increase the number of sites that provide this treatment option.

Corresponding Author: William Gunnar, MD, JD; Veterans Health Administration, Washington, DC, USA (e-mail: William.gunnar@va. gov).

\section{Compliance with Ethical Standards:}

Conflict of Interest: The author declares that he does not have a conflicts of interest.

\section{REFERENCES}

1. VA Office of Research and Development, VA Research on Obesity. Available at http://www.research.va.gov/topics/obesity.cfm. Accessed on 27 Dec 2016.

2. American Society for Metabolic and Bariatric Surgery, Estimate of Bariatric Surgery Numbers, 2011-2015. Available at https://asmbs.org/resources/ estimate-of-bariatric-surgery-numbers. Accessed on 27 Dec 2016.

3. Department of Veterans Affairs Unique Veterans Users Report FY 2014, Prepared by the National Center for Veterans Analysis and Statistics March 2016. Available at https://www.va.gov/vetdata/docs/ SpecialReports/Profile_of_Unique_Veteran_Users_2014.pdf. Accessed on 27 Dec 2016.

4. VHA Directive 1601, Non-VA Medical Care Program.

5. VHA Directive 1700, Veterans Choice Program. 